\title{
Neurobiology of developmental dyslexia Part 2: A review of magnetic resonance imaging (MRI) studies of the corpus callosum
}

\section{SO Wajuihian}

\section{Discipline of Optometry, University of KwaZulu-Natal, Private Bag X54001, Durban, 4000 South Africa}

Received 5 August 2011; revised version accepted 10 January 2012

<swajuihian@mweb.co.za>

\begin{abstract}
This paper forms part two of a review of the neurobiology of developmental dyslexia (DD) and here the focus is on magnetic resonance imaging (MRI) of the corpus callosum (CC) of dyslexic and nondyslexic subjects. The CC is a bundle of nerve fibres connecting the left and the right hemisphere of
\end{abstract}

the brain. Due to the role of this structure in interhemispheric transfer and integration between the hemispheres, the CC is significant in the search for the neurobiological basis of DD. (S Afr Optom 2012 71(1) 39-45)

Key Words: Developmental dyslexia, corpus callosum, magnetic resonance imaging (MRI), optometrist and dyslexia.

\section{Introduction}

Beside theories and studies associating anatomical deviations in brain structures-(asymmetry /symmetry) of the planum temporale (PT) to dyslexia ${ }^{1-3}$, another proposed mechanism in the neurobiological deviations in dyslexic brain is an abnormal collaboration between the brain hemispheres. The corpus callosum (CC) is a bundle of neural fibres beneath the cortex that connects the left and right cerebral hemispheres ${ }^{4-6}$ and it is the largest of the three commissures (bundles of nerve fibres that connect the two cerebral hemispheres) of the human brain, consisting of between 200 and 800 million axon fibres ${ }^{4-6}$. In providing a connection between homologous cortical areas, the $\mathrm{CC}$ functions primarily to facilitate inter-hemispheric communication between the two hemispheres and also plays an important role in the development of hemispheric asymmetry, cerebral dominance and laterality ${ }^{4-6}$.

The CC consists of three main anatomical parts.
The anterior part is called the genu, the posterior part is the splenium, between the genu and the splenum is the truncus, or body of the CC. The part between the body and the splenium is characteristically thinned and thus referred to as the isthmus. The rostrum is the part of the $\mathrm{CC}$ that projects posteriorly and inferiorly from the anterior-most genu ${ }^{4-5,7-8}$. The genu contains the highest density of thin myelinated axons whereas the splenium contains thick axons ${ }^{4-5}$. As the CC provides a connection between homologous cortical areas, the areas of CC has been shown to be "topographically organized with projections from specific cortical areas to specific callosal regions; anterior areas of cortex are connected through more anterior pathways", likewise for the posterior areas ${ }^{4,9}$. In a mid-sagittal cross-section (dividing the body into right and left passing through the midline) $)^{8,10}$, the macroscopic borders of the $\mathrm{CC}$ are not clearly defined, but microscopic techniques reveals myelinated axons with a relatively small diameter in the anterior and posterior third of the $\mathrm{CC}$ whereas fibers in the mid- 
body and posterior splenium contain thick fibers ${ }^{8}$. The $\mathrm{CC}$ is one of the anatomical structures of the human brain that is relatively easy to study in vivo ${ }^{8,11-12}$ and the sections can be subdivided into several functional and morphologically distinct sub-regions based on the topographical organization of cortical areas ${ }^{8,5}$. The main classification system used in the study of the $\mathrm{CC}$ is the Witelson's which defines five vertical callosal segments derived from dividing the $\mathrm{CC}$ along its anterior-posterior dimension and it is based on a general principle that (i) the anterior third of the $\mathrm{CC}$ contains fibres connecting the bilateral prefrontal cortices on either hemisphere and higher order sensory area (ii) the anterior and posterior mid-body (middle third) primarily contains crossing fibres for motor, somatosensory, and auditory cortices (iii) the splenium (posterior fifth) carries fibres mainly to temporal, parietal, and occipital lobes and (iv) the isthmus (in between the mid-body and splenium) is thought to carry fibres connecting perisylvian regions ${ }^{8,10}$.

The CC mediates its role in inter-hemispheric transfer and integration through either inhibition or excitation of its functions ${ }^{4}, 13$. The inhibitory function is a passive process and implies that $\mathrm{CC}$ performs its role by providing a pathway through which one hemisphere can inhibit the other thereby dominating a given function whereas the callosum's excitatory role in inter-hemispheric processing implies that the $\mathrm{CC}$ actively integrates its function of cerebral processing between the two hemispheres. Consequently, an increase in the connectivity between the hemispheres would decrease lateralization due to the excitatory qualities of the $\mathrm{CC}$ hemisphere ${ }^{4-5,13}$. Both inhibitory and excitatory messages have been demonstrated to pass through the $\mathrm{CC}^{4}$.

Apart from the principal role in inter-hemispheric integration, the $\mathrm{CC}$ plays an important role in the transmission of visual and auditory information in reading ${ }^{14}$. Reading is an acquired skill coordinated by a complex system ${ }^{15}$, which depends on the establishment of a facilitating neural circuit ${ }^{16}$. During reading, different brain areas are activated and the reading activity requires the flow of information from the posterior lobes to the frontal lobe of the brain ${ }^{8,17}$. In particular, word decoding (recognition) relies on the transfer of information between brain hemispheres; the left hemisphere processes information sequentially and specializes in linguistic processing whereas the right hemisphere (for most right handed persons) processes information in a holistic way and specializes in the identification of visual patterns ${ }^{18}$. Reading, a linguistic activity which engages both hemispheres and diverse areas of the brain requires efficient communication between these entities ${ }^{18}$. In multi-factorial conditions such as dyslexia, both genetic and environmental factors influence the establishment of this circuit and may result in faulty phonological awareness and a reading disability ${ }^{16}$, thus emphasizing the role of the $\mathrm{CC}$ in neural circuitry in reading.

The role of the $\mathrm{CC}$ in reading disorder has been well-documented in the literature ${ }^{19-27}$. Temple et al (cited by Galaburda) ${ }^{19}$ suggested that congenital abnormalities in the $\mathrm{CC}$ are associated with reading disorders. More so, it has been demonstrated that defective inter-hemispheric integration and transfer of information is associated with reading disorders $20-21$ and that some children with learning disabilities (LD) also display neurological signs suggestive of interhemispheric disconnection ${ }^{22}$. Another perspective is that autopsy studies ${ }^{23-24}$, as well as neuro-imaging studies $^{25-26}$, have revealed symmetry and an enlargement of the PT, a language-relevant cortical structure in the brain. The presence of enlarged PT associated with symmetrical PT was suggested to be due to reduced cell death during fetal development which then results in an increased number of axons passing through the $\mathrm{CC}^{27}$. The resultant larger callosal area however, precludes a high capacity for interhemispheric transfer of information to the perisylvian-language regions due to the presence of ectopias (misplaced neural cells) $)^{6,23-24,27 .}$

Overall, due to its principal role of inter-hemispheric transmission and integration of information and specifically its role in transmission of visual and auditory information in reading 24 , the $\mathrm{CC}$ has been an area of intense research in the quest for the neurobiological substrate of DD. In this paper, MRI studies of the $\mathrm{CC}$ of dyslexic and normal readers are reviewed to address the question of whether anatomical deviations (morphology of the $\mathrm{CC}$ in this case) distinguish the dyslexic and non-dyslexic brain.

\section{A review of magnetic resonance imaging of the corpus callosum}

Magnetic resonance imaging studies on the $\mathrm{CC}$ 
have compared the relative size, shape or subdivisions of the $\mathrm{CC}$ between dyslexics and control.

\section{Findings on the Genu}

Hynd et al12, in 1995, examined the morphology of the corpus callosum in 16 dyslexic (Mean age 116.50 months) and 16 control children (Mean age 132.75 months) matched by gender. Using a midsagittal scan they obtained area measurements for five regions of the $\mathrm{CC}$, although only the results for the genu and splenium were reported. Significant differences between dyslexic and the normal-reading group were found only in the genu, which was smaller in the dyslexic children. The authors also reported a moderate correlation between reading achievement and the relative size of the genu and splenium, which led to the conclusion that successful reading acquisition is associated with a larger CC particularly in the posterior and anterior regions ${ }^{22}$.

\section{Findings on the Splenium}

Two studies ${ }^{28-29}$ reported enlarged spleniums in the dyslexic group. First, Duara et a ${ }^{28}$ measured the area of subdivisions of the $\mathrm{CC}$ on a midsagittal brain section, obtained from 21 dyslexic (Mean age $39.1 \pm 11.0$ years) and 29 control subjects (Mean age 35.3 \pm 10.0 ). Dyslexic subjects had larger spleniums than non-dyslexic subjects, and dyslexic female subjects had larger spleniums than dyslexic male subjects ${ }^{28}$. Furthermore, both the genu area and the $\mathrm{CC}$ in general were found to be larger in female than in male dyslexic adults ${ }^{2}$. Rumsey et a ${ }^{29}$ measured the anterior, middle, and posterior regions of the $\mathrm{CC}$ of 21 dyslexic men (Mean age $27 \pm 5$ years) and 19 control (Mean age $27 \pm 6$ years). Using the "whole corpus callosum as a covariate", the authors found that the area of the posterior portion of the CC-roughly corresponding to the splenium and the isthmus was significantly enlarged in dyslexics relative to control subjects (one-tailed $p=0.01)$ and that neither the anterior nor the middle portion of this structure differed between groups.

\section{Findings on the total callosal area}

Several studies $6,14,22,28,30-31$ reported differences in total callosal area or shape/length of the $\mathrm{CC}$ but only two ${ }^{6,14}$ found the $\mathrm{CC}$ to be shorter in dyslexics. First, the study by Von Plessen et al ${ }^{14}$ compared both shape and size of the $\mathrm{CC}$ and its sub regions be- tween 20 right-handed boys with DD matched in age (Mean age 142 months), gender and handedness with 20 right-handed boys (Mean age 141months) as control. The authors reported that shape analysis revealed shorter CC shape in the dyslexic group, localized in the posterior isthmus region but there were no significant group differences in overall $\mathrm{CC}$ area/size or sub- regions ${ }^{14}$. This region contains inter-hemispheric fibres from primary and secondary auditory cortices. The authors ${ }^{14}$ claimed that the shape abnormality was confirmed by an automatic classification procedure with an accuracy of $78 \%$ over the entire sample and that a shape length difference larger than a fixed threshold in the posterior mid-body region could correctly discriminate between control and dyslexic subjects. It was also noted that dyslexics presumably fail to undergo the massive myelination in this region during reading acquisition ${ }^{14}$. The second study in 2008 that found shorter $\mathrm{CC}$ in dyslexics was reported by Duta et $a l^{6}$. The authors investigated the extent to which the shape of the $\mathrm{CC}$ can predict the presence of dyslexia. Their statistical shape analysis revealed differences in $\mathrm{CC}$ on the length of the isthmus region between a group of 20 normal (Mean age $35.3 \pm 10.0$ years) and 20 dyslexic subjects (Mean age $39.1 \pm 11.0$ years). The dyslexic CC shapes (in the isthmus) was found to be shorter than normal. Duta et al6 concluded that their study was the first to attempt to automatically predict dyslexia based on the characteristics of the CC extracted from MR images.

Other studies $16,22,28,30-31$ that reported on the total callosal size found increased sizes of the CC. In 2010, Casanova et $a l^{16}$, using a novel pattern recognition technique that offers a point-by-point shape descriptor of the CC examined 16 dyslexic men (Mean age 28.2 years) and 14 age-matched controls (Mean age 25.1 years). The study revealed a generalized increase in the size of the $\mathrm{CC}$ in dyslexia with a concomitant diminution at its rostral and caudal poles. Duara et $a^{28}$ found the $\mathrm{CC}$ in general were larger in female than in male dyslexic adults. Njiokiktjien et al ${ }^{22}$ studied callosal size in children with LD which included 39 dyslexic (age range was 2.5 to 14 years) and 42 control subjects (age range 0 to 20 years). The authors $^{22}$ found that children with familial dyslexia, had a thicker CC, which they claimed was an indication of poorly understood neuro-developmental mechanism that inhibits the establishment of cerebral dominance. 
Robichon and Habib ${ }^{30}$ studied CC morpohology in 16 dyslexic adults and 12 controls using a high resolution brain MRI scan. The two groups differed remarkably with respect to the shape and regional size of the $\mathrm{CC}$, with the dyslexic subjects exhibiting a more circular and rounded thicker callosal shape than the control subjects ${ }^{30}$. Secondly, the mid-sagittal surface is on the average larger than in controls, in particular in the isthmus ${ }^{30}$. Also, Robichon et $a^{31}$ in 2000 used a novel method based upon the measurement of four angles, analyzed the CC of 23 adult male dyslexics $(24.47 \pm 5.68)$ and 25 age-matched controls (Mean age 26.82 years) on MRI sagittal scans. Two out of the four angles measured showed significant differences between the groups that were consistent with previous findings ${ }^{30}$ concerning the size of the $\mathrm{CC}$ in dyslexics. In particular, posterior regions indicated a lowered situated $\mathrm{CC}$ in dyslexics ${ }^{31}$.

Four studies $29,32-34$ found no difference in total callosal area. First, Pennington et al ${ }^{32}$ in a study that controlled for age, gender, ADH and intelligence quotient, examined brain structure differences in dyslexic and control subjects. The subjects comprised 75 individuals with reading disability (RD) (Mean age 17.43 \pm 4.29 ) and 22 control subjects without RD each a single member of a twin pair. The authors found no difference in any part of the $\mathrm{CC}$ between the dyslexic and control group. Cassanova et al ${ }^{33}$ studied brain size in 16 dyslexics and 14 control. The authors found no differences in the cross-sectional area of the CC in dyslexics and control. Larsen et al ${ }^{34}$ found no differences in the total callosal area or the splenium in male sample of 19 dyslexic adolescents and 17 normal readers. The authors also reported no difference in size of the $\mathrm{CC}$ in sub-groups of dyslexia related to reading profile or symmetry. Similarly, Rumsey et $a l^{29}$ found no difference in relative $\mathrm{CC}$ area.

\section{Discussion}

A direct comparison and synthesis of the studies on CC morphology is complicated due to considerable variations in methods (especially characteristic of participants) and diverse findings across studies. Only the study by Hynd et al ${ }^{12}$ found the genu to be smaller in the dyslexic children. The authors argued that their findings of a reduced genu may be due to the bilateral frontal cytoarchitectonic anomalies found in the brains of dyslexic individuals. Duara et $a l^{28}$ and Rumsey et $a l^{29}$ reported the spleniums to be enlarged in the dyslexics compared to controls. Although the studies by Duara et $a l^{28}$ and Rumsey et a ${ }^{29}$ reported similar findings of enlarged spleniums in the dyslexic group, both studies used different mid-sagittal slice thickness (Duara et al ${ }^{28}=7 \mathrm{~mm}$, Rumsey et al ${ }^{29}=5$ $\mathrm{mm}$ ) as well as varying gender and age ratios (see table). More so, in the study by Duara et a ${ }^{28}$ co-diagnostic variables such as attention deficit hyperactivity disorder was not controlled. In the discussion of their findings, Rumsey et a ${ }^{29}$ suggested that the increased area of the posterior CC may reflect anatomical variations associated with deficient lateralization of function in posterior language regions of the cortex. The splenium which comprises part of the posterior third of the $\mathrm{CC}$ contain fibres from the temporal and posterior parietal cortex, as well as from occipital cortex ${ }^{4}, 8,10$. Specifically, the splenium may be considered the most critical region because it contains axons linking the PT and the angular gyrus ${ }^{35}$. Also, as the reading process is a complex task that relies on the brain systems in the posterior, parieto-temporal, and occipital brain regions in the left hemisphere ${ }^{36}$, the findings of enlarged spleniums therefore may be relevant to the etiology of dyslexia as this region contains fibres from the temporal and posterior parietal cortex, as well as from occipital cortex which is associated with posterior language regions ${ }^{29,} 36-37$ the same region where unusual anatomical asymmetries have been reported in magnetic resonance imaging $28-29,34,38$.

Duta et $a l^{6}$ and Von Plessen et al ${ }^{14}$ found shorter $\mathrm{CC}$ although there are also some differences in the subjects' characteristics between the two studies. As the posterior $\mathrm{CC}$ undergoes a massive myelination in normal readers during the years of reading acquisition $^{14}$, Von Plassen et al ${ }^{14}$ suggested that in their study, shape analysis indicates that the $\mathrm{CC}$ in the dyslexic group fails to undergo that development, which then resulted in a shorter $\mathrm{CC}$, a finding which they argued, corroborates the findings of Castro-Caldas et $a l^{39}$ who studied the influence of learning to read and write on the morphology of the CC in illiterate and literate subjects and found significant differences in the posterior mid-body section with a thinner CC in the illiterate group. Thus, Von Plessen et al ${ }^{14}$ suggested that fibres that are thought to cross over their measured region of interests in the posterior mid-body, the 
region adjacent to the isthmus area are the sensorimotor ones as well as fibres from the superior and posterior parietal region and from the superior temporal region. Temporal and parietal lobe regions are crucial for the development of language and language processing, since they contain the primary, secondary and tertiary auditory cortical areas ${ }^{14}$.

Three studies ${ }^{16,28,30}$ found larger $\mathrm{CC}$ in the dyslexic population. Also, there are some methodological variations among the studies. Overall, enlarged CC may suggest that the $\mathrm{CC}$ of dyslexics have failed to undergo the usual developmental evolution that normally gives the typical shape of the $\mathrm{CC}^{30}$. This shaping process presumably occurs during the peri-natal period and the first post-gestational weeks and would result from conspicuous loss of axons during this critical period. Subsequently, the callosal area will increase gradually to adulthood, probably reflecting the progressive myelination of persisting fibres ${ }^{30}$ which then implies that larger CC in dyslexics would reflect incomplete expression of these regressive events ${ }^{30}$. However, the finding of smaller CC in some dyslexic brains ${ }^{12}$ is not consistent with this hypothesis. An enlarged CC could also result from some hereditary brain mechanisms ${ }^{22}$. In addition, a large $\mathrm{CC}$ might develop prenatally if cell death does not occur and if an excess of callosal neurons cross the midline ${ }^{22}$.

Taken together, the contradictory findings in the MRI results on the $\mathrm{CC}$ may be due to some methodological differences. Specifically, methodological variations in $\mathrm{CC}$ studies may be due to the following factors:

- $\quad$ effect of subjects placement within the scanner and differences in the orientation of the scan plane used to generate the mid-sagittal image may result in variability in appearance and area measurements of the callosum ${ }^{40-41}$

- difficulty determining a proper mid-sagittal section 6,11

- the possible influence of the brain volume on estimated $\mathrm{CC}$ area as well as differences in methods for segmentation and subdivision of the $\mathrm{CC}^{6}$

- inadequate control for independent variables such as gender, brain size, socioeconomic status, cognitive variables, handedness, age and diagno$\operatorname{sis}^{11}$

- the methods for subdivision of the $\mathrm{CC}$ have also differed between studies. Some studies on CC morphology relied on Witelson's classification of $1989^{5,8}$ whereas a marked difference were reported by Hofer ${ }^{8}$ in the mid-body and anterior third of the CC. In particular, callosal motor fibres bundles were found to cross the $\mathrm{CC}$ in a more posterior location than previously indicated ${ }^{6}$. Therefore, it is unreliable to compare results directly when methods for subdivisions are different.

In addition to variables associated with differences in methodologies, other factors which may contribute to differences in $\mathrm{CC}$ measurements across studies are: Firstly, the $\mathrm{CC}$ is the main axonal pathway in the brain but there are additional commissures in the brain. The functions of these sub- cortical commissures may be confounding in these studies on the CC morphology and its role in inter-hemispheric transfer of information ${ }^{4}$. In addition, brain morphology is influenced by genetic and environmental factors ${ }^{14,39,42}$ resulting in an impaired growth and function of the part of the CC containing auditory fibres which would result in a dysfunction of phonological decoding ${ }^{14,42}$. Environmental factors that may affect the developmental abnormality of the posterior mid-body of the CC could arise from lacking stimulation due to delayed reading acquisition $14,39,42$.

From a neuro-developmental point of view, differences in callosal size may reflect hormonal influences during critical periods of development of inter-hemispheric connections ${ }^{43}$. Specifically, testosterone has been considered an etiological factor in CC development and may influence callosal morphology by altering naturally occurring neural regressive events in the perisylvian regions of the neocortex ${ }^{44}$. Low levels of testosterone has been hypothesized to reduce axon elimination, resulting in a larger isthmus of the $\mathrm{CC}$ and a subsequent reduction in functional brain asymmetry and laterization, a hypothesis supported by evidence including findings of gender and handedness differences in CC morphology 45 and by the observation that the $\mathrm{CC}$ may be larger in homosexual males in whom lower than normal androgen levels during early development have been hypothesized ${ }^{44}$. Furthermore, callosal transmission may be affected by defects in the fibres of the $\mathrm{CC}$ as well as by anomalies in the cells in the region of origin ${ }^{12,20}$. Also, size variations 
in the CC may reflect differences in the number or size of axons that connect these regions, an increase in extra-cellular and vascular space. Changes may also indicate differences in the number of cortical neurons within homologous regions ${ }^{12,20}$. Invariably, an abnormally thick CC would inhibit right hemisphere competence and impair reading $22,44,46-48$.

\section{Summary, conclusion and recommendations}

The review of studies comparing the $\mathrm{CC}$ of dyslexic and normal readers yield contradictory results. In summary, eleven studies have measured in vivo the size or shape of the corpus callosum in dyslexic participants compared to control group. One study ${ }^{12}$ found smaller genu in dyslexics, two studies ${ }^{28-29}$ reported enlarged splenium in dyslexics and two studies $^{6,14}$ reported shorter CC in dyslexics. Also, five studies $^{16,22,28,30-31}$ reported increase in the size of the $\mathrm{CC}$ in the dyslexics whereas three studies ${ }^{32-34}$ found no difference in callosal size between dyslexic and control.

In conclusion, although some studies found a difference in the morphology of the $\mathrm{CC}$ between dyslexic and control, there is no consistent evidence to fully establish a link between CC morphology and dyslexia. The main limitation with studies on $\mathrm{CC}$ morphology is the lack of consensus on the methodological approach. Therefore, more research, possibly collaborative research from different laboratories, among experts is needed so that documented sources of variations can be controlled. This approach will better clarify whether there are differences in CC morphology between dyslexic and normal readers. Specifically, studies should be designed to simultaneously examine the effects of independent variables such as gender, brain size, socioeconomic status, cognitive variables, handedness, age and diagnosis ${ }^{11}$. In addition, measurement techniques should be simple enough to allow rapid collection of data in many subjects considering that studies of biological factors influencing human brain structure require large samples $^{7,11}$. Finally, although the $\mathrm{CC}$ is the main axonal pathway connecting both hemisphere of the brain ${ }^{4}$, it will be necessary to consider the roles of sub-cortical commissures in inter-hemispheric transfer.

This review is intended to enhance our understanding of the neurobiology of developmental dyslexia.

\section{Acknowledgement}

I am grateful to Mr Percy Mashige and Dr Chris Isike for comments on the final draft of this manuscript.

\section{References}

1. Galaburda AM, Sherman GE, Rosen GD, Aboitiz F, Geschwind N. Developmental dyslexia: Four consecutive patients with cortical anomalies. Ann Neurol 198518 222233.

2. Humphreys P, Kaufmann W, Galaburda AM. Developmental dyslexia in women: Neuropathological findings in three patients. Ann Neurol 199028 727-738.

3. Livingstone M, Rosen G, Drislane F, Galaburda AM. Physiological and anatomical evidence for a magnocellular defect in developmental dyslexia. Proceed Nat Acad Sci 1991 88 7943-7947.

4. Bloom JS, Hynd GW. The role of the corpus callosum in interhemispheric transfer of information: Excitation or inhibition? Neuropsychol Rev 200515 59-71.

5. Van der Knaap LJ, van der Ham IJM. How does the corpus callosum mediate interhemispheric transfer? A review. Behav Brain Res 2011 doi:10.1016/j.bbr.2011.04.018.

6. Duta N, Lundervold A, von Plessen K, Klauschen F, Jain AK. Predicting dyslexia based on the shape of the Corpus Callosum in MR images. Presented at the 15th International Conference on Pattern Recognition, September 3-8, 2000, Barcelona, Spain.

7. Beaton, AA. The Neurobiology of Dyslexia. In: Turner M, Rack J. The study of dyslexia New York: Kluwer Academic Press 2004 pp 46-76.

8. Hofer S, Frahm J. Topography of the human corpus callosum revisited-Comprehensive fibres tractography using diffusion tensor magnetic resonance imaging. Neuroimage 200632 989-994

9. Heim S, Keil A. Large-scale neural correlates of developmental dyslexia. Europ Child \&Adol Psychiat 200413125 140.

10. Kochunov P, Lancaster J, Hardies J,Thompson PM Woods RP, Cody JD. Mapping structural differences of the corpus callosum in individuals with $18 \mathrm{q}$ deletions using targetless regional spatial normalization. Human Brain Mapping 200524 325-331.

11. Leonard CM. Corpus Callosum: Sex or Size? Cerebral Cortex 199772.

12. Hynd GW, Hall J, Novey ES, Eliopulos D, Kathryn Black K, Jose J. Gonzalez, JJ Edmonds J, Riccio C, Cohen M. Dyslexia and corpus callosum morphology. Arch Neurol 199552 32-38.

13. Yazgan MY, Wexler BE, Kinsbourne M, Functional significance of individual variations in callosal area. Neuropsychologica 199533 769-779.

14. Von Plessen K, Lundervold A, Duta N, Heiervang E, 
Klauschen F, Smievoll A, Ersland L Hugdahl K. Less developed corpus callosum in dyslexic subjects-A structural MRI study. Neuropsychol 200240 1035-1044.

15. Wajuihian SO. Neurobiology of developmental dyslexia. Part 1: A review of evidence from autopsy and structural neuro-imaging studies. S Afr Optom 201170 184-195.

16. Casanova MF, El-Baz A, Elnakib A, Giedd J, Rumsey JM, Williams EL, Switala AE. Corpus Callosum shape analysis with application to dyslexia. Transl Neurosc 20102124 130.

17. Shaywitz SE, Mody M, Shaywitz BA. Neural mechanisms in dyslexia. Current directions Psychol Sci 15 278-281.

18. The Sage handbook of dyslexia. Edited by Reid G, Fawcett A, Frank Manis F, Siegel L. Sage Publications; Los Angeles, 2008

19. Galaburda AM. Neurology of developmental dyslexia. Curr Opinion Neurobiol 19933 237-242.

20. Fabbro F, Pesenti S, Facoetti A, Bonanomi M, Libera L, Lorusso ML. Callosal transfer in different subtypes of developmental dyslexia. Cortex 200137 65-73.

21. Markee T, Brown WS, Moore LH, Theberge DC. Callosal function in dyslexia: Evoked potential interhemispheric transfer time and bilateral field advantage. Develop Neuropsych 199612 409-428 (Abstract).

22. Njiokiktjien C, De Sonneville L, Vaal J. Callosal size in children with learning disabilities Behav Brain Researh 199464 213-218.

23. Galaburda AM. Ordinary and extra-ordinary brain development. Anatomical variations in developmental dyslexia. Ann Dyslexia 198939 67-80.

24. Galaburda AM. Developmental dyslexia: Multilevel syndrome. Dyslexia 19995 183-191.

25. Larsen JP, Høien T, Lundberg I, Ødegaard H. MRI evaluation of the size and symmetry of the planum temporale in adolescents with developmental dyslexia. Brain and Lang 199039 289-301.

26. Hynd GW, Semrud-Clikeman M, Lorys AR, Novey ES, Eliopulos D. Brain morphology in developmental dyslexia and attention deficit disorder/hyperactivity. Arch Neurol 1990 47 919-926.

27. Galaburda AM. Dyslexia: A molecular disorder of neuronal migration. Ann Dyslexia 200555 151-165.

28. Duara R, Kushch A, Gross-Glenn K, Barker WW, Jallad B, Pascal S, Loewenstein DA, Sheldon J, Rabin M, Levin B, Lubs H. Neuroanatomic differences between dyslexic and normal readers on magnetic resonance imaging scans. Arch Neurol 199148 410-416.

29. Rumsey JM, Casanova M, Mannheim GB. Corpus callosum morphology as measured with MRI, in dyslexic men. Biol Psychiatry 199639 769-775.

30. Robichon F, Habib M. Abnormal callosal morphology in male adult dyslexics: Relationships to handedness and phonological abilities. Br Lang 199862 127-146.

31. Robichon F, Bouchard P, Démonet J, Habib M. Developmental dyslexia: Re-evaluation of the corpus callosum in male adults. Eur Neurol 200043 233-237.

32. Pennington BF, Filipek PA, Lefly D, Churchwel J, Galaburda A, Alarcon M DeFries JC. Brain morphometry in reading-disabled twins. Neurology 199953 723-729.
33. Casanova MF, Araque J, Giedd J. Reduced brain size and gyrification in the brains of dyslexic patients. $J$ Child $\mathrm{Neu}$ rol 200419 275-281.

34. Larsen JP, Hoien T, Odegaard H. Magnetic resonance imaging of the corpus callosum in developmental dyslexia. Cogn Neuropsychol 1992 9123-134.

35. Semrud-Clikeman M. Evidence from imaging on the relationship between brain structure and developmental language disorders. Sem Ped Neur 19974 117-124.

36. Caylak E. Neurobiological approaches on brains of children with dyslexia Acad Radiol 200916 1003-1024.

37. Galaburda AM. Developmental dyslexia and animal studies: at the interface between cognition and neurology. Cognition 199450 133-149.

38. Kusch on PT Kushch A, Gross-Glenn K, Jallad B, Lubs H, Rabin M, Feldman E, Duara R. Temporal lobe surface area measurements on MRI in normal and dyslexic readers. Neuropsychol 199331 811-821.

39. Castro-Caldas A, Miranda PC, Carmo I, Reis A, Leote F, Ribeiro C. Influence of learning to read and write on the morphology of the corpus callosum. Euro J Neurol 19996 23-28.

40. Mitchell TN, Free SM, Merschhemke M, Lemieux L, Sisodiya SM, Shorvon SD. Reliable callosal measurement: Population normative data confirm sex-related differences. Am J Neuroradiol 200324 410-418.

41. Rauch and Jenkins Rauch RA, Jinkins JR. Variability of corpus callosal area measurements from midsagittal magnetic resonance images: Effect of subject placement within the scanner. Am J Neuro-Radiol 199617 27-28.

42. Jäncke L, Steinmetz H. Brain size: a possible source of interindividual variability in corpus callosum morphology In: The role of the human corpus callosum in sensory motor integration: anatomy, physiology, and behavior; individual differences and clinical applications, edited by E. Zaidel, M. Iacoboni, Pacual-Leone AP, New York: Plenum Press, 1998.

43. Habib M. The neurological basis of developmental dyslexia. An overview and working hypothesis. Brain 2000123 2373-2399.

44. Moffat SD, Hampson E, Wickett JC, Philip A. Vernon PA, Lee DH . Testosterone is correlated with regional morphology of the human corpus callosum Brain Research 1997 767 297-304.

45. Burke, HL, Yeo, RA Systematic variations in callosal morphology: The effects of age, gender, hand preference, and anatomic asymmetry. Neuropsychol 19948 563-571.

46. Seidman LJ, Valera EM, Makris N. Structural brain imaging of attention-deficit/hyperactivity disorder Biol Psychiat 200557 1263-1272.

47. Zaidel E, Marco I. The parallel brain: The cognitive neuroscience of the corpus callosum. The MIT Press Cambridge, Massachusetts, 2003.

48. Schlaug G, Jancke L, Huang Y, Staigeri J, Steinmetz H. Increased corpus callosum size in musicians. Neuropsychologia 199533 1047-1055. 